\title{
Resistance assessments of steel columns of variable cross sections
}

\author{
P. Błażejewski, S. Kołodziej, J. Marcinowski \& V. Sakharov \\ University of Zielona Góra, Zielona Góra, Poland
}

\begin{abstract}
Steel columns of hollow cross-sections and simply supported at their ends are considered in the paper. Columns of such shapes are recently frequently proposed by architects in public utility buildings of various types. In this work authors present the comparatively simple procedure which can be used by designers of steel columns of such shapes. The value of critical force required in the procedure was derived for columns of variable cross section by means of Mathematica ${ }^{\mathrm{TM}}$ system. The closed formulae were obtained for a rod with a certain, predefined geometry being the surface of revolution. Critical forces obtained by means of derived formulae were compared with numerical solutions. To assess the compression resistance of considered rods the general Ayrton-Perry approach was applied and bow imperfection with assumed amplitude was used in the analysis. Examples inserted in the paper confirm the correctness and the effectiveness of the proposed procedure.
\end{abstract}

\section{INTRODUCTION}

Tapered columns resembling a spindle shape and fabricated as a steel, hollow sections are more and more often encountered in engineering practice (Figure 1). Some of the main reasons for their increasing use are the material savings, a greater load capacity and aesthetic requirements (Thompson et al. 2007, Marcinowski 2015, Marcinowski \& Sadowski 2020). Provisions of EN 1993 (2005) do not deliver direct procedures which can be used in designing of such kind of columns. Existing design procedures based on general Ayrton \& Perry (1886) approach refers only to columns of constant sections. This general approach requires knowledge of the critical force value and it is the principal problem which should be solved.

There are a large number of papers in which the buckling problem of non-prismatic columns is considered (Li et al. 1995, Le 2001, Elishakoff \& Rollot 1999). Value of the critical forces is absolutely necessary to the assessment of load bearing capacity.

In the present paper the effective method of determination of the critical force has been presented. To this end the energetic criterion of stability proposed in Timoshenko \& Gere (1961) was used. The obtained analytical expression on the critical force has been verified positively by means of numerical solutions using commercial softwares based on finite element method.

To assess the compression resistance of the considered kind of columns the general AyrtonPerry approach is adopted. This approach dating from XIX century (Ayrton \& Perry 1886), after many modifications, was inserted into contemporary design provisions (EN 19931-1:2005) and used by many authors (Szalaj \& Papp 2010; Szalaj 2017; Giżejowski et al. 2019).

The stress criterion, in which the maximum stress was equated to the yield stress $f_{\mathrm{y}}$ in the most stressed section, was the condition from which the allowable compressive force was obtained. Similarly as in the Ayrton-Perry approach, the initial bow imperfection has been taken into account with the amplitude corresponding to the fabrication quality class. The effect of the eccentricity amplification, typical for slender compressed members, was taken into account as well. The stress condition has been checked in every section $\mathrm{x}$ along the 


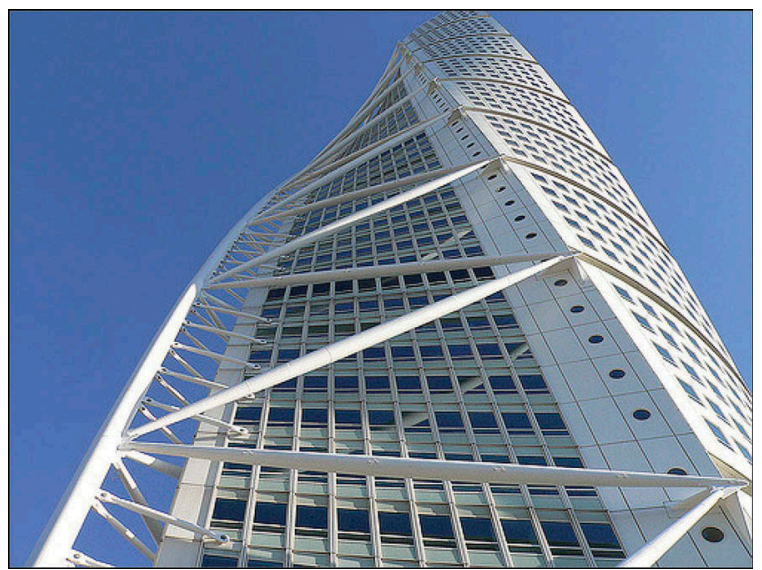

Figure 1. Santiago Calatrava's „Turning Torso” in Malmö.

column axis because, due to the column cross-section variability, it was not possible to predict in advance in which cross-section the stress condition will be decisive.

Only specific class of geometries of bulged column was considered in the paper. The effectiveness of the proposed designing procedure was illustrated on examples. They confirm the effectiveness of the proposed approach.

In authors' opinion the presented procedure can be used by engineers designing steel, bulged columns of the shape considered in the paper.

\section{DERIVATION OF FORMULAE FOR THE CRITICAL FORCE}

Due to the fact that columns of variable cross-sections are subject of interest in this work, the critical force can be established only in an approximate way using the energetic criterion of stability (Timoshenko \& Gere 1961). According to this criterion the critical force for pin ended column can be obtained from the formula:

$$
P_{k r}=E \cdot \int_{0}^{L} w^{\prime 2} d x / \int_{0}^{L} \frac{w^{2}}{J(x)} d x
$$

in which $w(\mathrm{x})$ is expected buckling form adopting here the shape of $1 / 2$ wave of sine function with the amplitude $A_{0}$ (Figure 2). In the equation (1) $J(\mathrm{x})$ is the moment of inertia of the cross section and $E$ is the Young's modulus. The moment of inertia $J(\mathrm{x})$ is defined in the following way (Figure 3):

$$
J(x)=\frac{\pi}{4}\left[r(x)^{4}-(r(x)-t)^{4}\right]
$$

in which $r(\mathrm{x})$ is the external radius of variable cross section defined as follows:

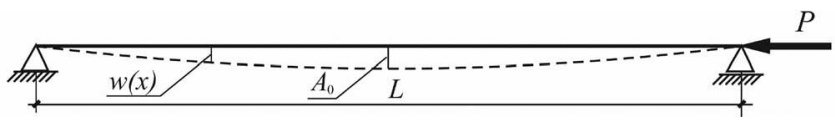

Figure 2. Buckling mode of the pin ended compressed bar. 
a)

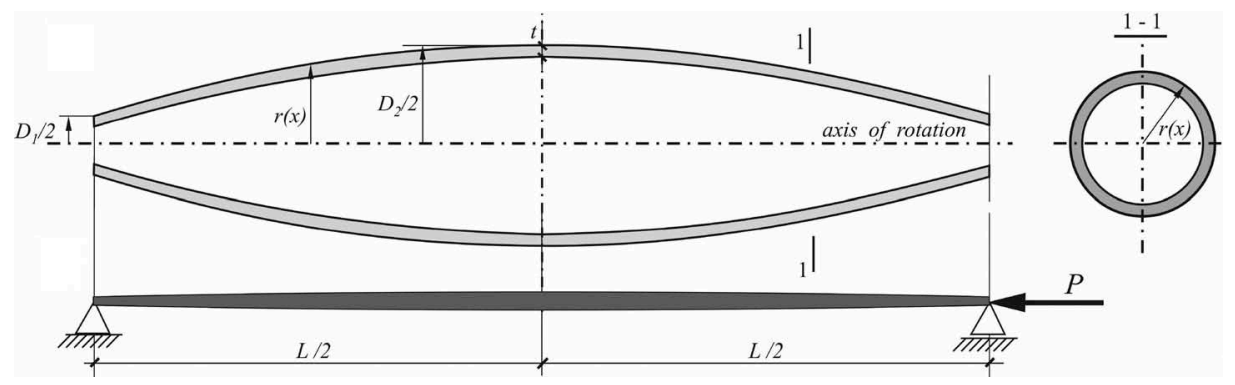

Figure 3. Sections of the rod a) (scale not preserved) and static scheme of the compressed bar b).

Table 1. Derived formulae on critical forces $P_{k r} /\left(E \cdot L^{2}\right)$.

\begin{tabular}{llll}
\hline $\mathrm{D}_{2} / \mathrm{L}$ & $\mathrm{t} / \mathrm{L}=1 / 1000$ & $\mathrm{t} / \mathrm{L}=2 / 1000$ & $\mathrm{t} / \mathrm{L}=3 / 1000$ \\
\hline $4 / 200$ & $5.20843 \cdot 10^{-7}$ & $3.10434 \cdot 10^{-7}$ & $2.46778 \cdot 10^{-7}$ \\
$5 / 200$ & $2.85456 \cdot 10^{-7}$ & $1.65675 \cdot 10^{-7}$ & $1.28347 \cdot 10^{-7}$ \\
$6 / 200$ & $1.75025 \cdot 10^{-7}$ & $9.97776 \cdot 10^{-6}$ & $7.59508 \cdot 10^{-6}$ \\
$7 / 200$ & $1.15857 \cdot 10^{-7}$ & $6.51976 \cdot 10^{-6}$ & $4.89997 \cdot 10^{-6}$ \\
$8 / 200$ & $8.10812 \cdot 10^{-6}$ & $4.51815 \cdot 10^{-6}$ & $3.36286 \cdot 10^{-6}$ \\
\hline
\end{tabular}

$$
r(x)=\frac{D_{1}}{2}+\left(\frac{D_{2}}{2}-\frac{D_{1}}{2}\right) \sin \frac{\pi x}{L} .
$$

Further considerations presented in this work were limited to some specific geometry defined uniquely by the column's length $L$. It was assumed that the external diameter of the terminal cross section is constant and equal $D_{1}=1 / 100 \mathrm{~L}$. External diameters of the middle cross sections of considered bars adopt the following values $D_{2} / \mathrm{L}=4 / 200,5 / 200$, $6 / 200,7 / 200,8 / 200$. The wall thickness $t$ is constant and adopts the following values: $t / L=1 / 1000,2 / 1000,3 / 1000$. These particular geometrical parameters were used in further considerations.

Formulae expressing the critical forces for particular geometries of columns are presented in Table 1. They were obtained from eqn. (1) by means of derivations carried out in Mathematica $^{\text {TM }}$ system (Wolfram 1988).

\section{NUMERICAL VERIFICATION OF DERIVED FORMULAE ON CRITICAL FORCES}

The correctness of derived formulae were verified numerically by means of commercial software based on finite element method. Three different programs were used and two different models were created. The beam model was prepared in Autodesk ${ }^{\circledR}$ Robot $^{\mathrm{TM}}$ Structural Analysis Professional system (RSA 2016), the shell model was created in COSMOS/M system (COSMOS/M 2002) while the 3D model was prepared in Simulia Abaqus system (Abaqus 2020).

In the performed comparative analysis the following data were adopted: $L=1000 \mathrm{~mm}, D_{1}=L /$ $100=10 \mathrm{~mm}, E=210000 \mathrm{MPa}$. Results are presented in Table 2.

Results presented in Table 2 confirm quite good correspondence between results obtained by means of derived formulae (labelled as Analytical) and results obtained numerically for the beam model (ROBOT), for the shell model (COSMOS/M) and for the 3D model (ABAQUS). 
Table 2. Critical forces in $[\mathrm{kN}]$ obtained for the column of length $L=1000 \mathrm{~mm}$.

\begin{tabular}{lllllll}
\hline $\mathrm{t}[\mathrm{mm}]$ & Calculation & $\mathrm{D}_{2}=20 \mathrm{~mm}$ & $\mathrm{D}_{2}=25 \mathrm{~mm}$ & $\mathrm{D}_{2}=30 \mathrm{~mm}$ & $\mathrm{D}_{2}=35 \mathrm{~mm}$ & $\mathrm{D}_{2}=40 \mathrm{~mm}$ \\
\hline 1 & Analytical & 4.032 & 7.357 & 11.998 & 18.126 & 25.900 \\
1 & ROBOT & 4.139 & 7.564 & 12.301 & 18.470 & 26.418 \\
1 & COSMOS & 4.096 & 7.561 & 12.300 & 18.469 & 26.162 \\
1 & ABAQUS & 4.158 & 7.561 & 12.373 & 18.584 & 26.364 \\
\hline 2 & Analytical & 6.765 & 12.675 & 21.047 & 32.210 & 46.479 \\
2 & ROBOT & 6.896 & 12.955 & 21.441 & 32.581 & 47.155 \\
2 & COSMOS & 6.822 & 12.946 & 21.438 & 32.581 & 46.549 \\
2 & ABAQUS & 6.946 & 13.032 & 21.678 & 32.931 & 47.095 \\
\hline 3 & Analytical & 8.510 & 16.362 & 27.649 & 42.857 & 62.447 \\
3 & ROBOT & 8.538 & 16.506 & 27.816 & 42.780 & 62.936 \\
3 & COSMOS & 8.438 & 16.488 & 27.808 & 42.780 & 61.639 \\
3 & ABAQUS & 8.748 & 16.819 & 28.424 & 43.647 & 62.897 \\
\hline
\end{tabular}

\section{RESISTANCE OF THE COMPRESSED COLUMN}

The resistance of the considered rod will be assessed on the basis of classical Ayrton-Perry's approach. Let us assume that the rod has an initial bow imperfection with amplitude $e_{0}$ (Figure 4) in a form of the one half-wave sine function defined as follows:

$$
e(x)=e_{0} \sin \frac{\pi x}{L}
$$

The total deflection $u_{c}(x)$ can be obtained from the formula (cf. Timoshenko \& Gere 1961):

$$
u_{c}(x)=f_{c} \sin \frac{\pi x}{L}, f_{c}=\frac{e_{0}}{1-\frac{P}{P_{k r}}}
$$

where $f_{c}$ - total eccentricity at middle section of the rod, $P_{k r}$ - critical buckling force.

The maximum longitudinal stresses at arbitrary cross section defined by $x$ can be calculated from the formula:

$$
\sigma_{\max }=\frac{P}{A(x)}+\frac{P f_{c}}{W(x)} \sin \frac{\pi x}{L} \leq f_{y}
$$

in which:

$$
A(x)=\pi\left[r(x)^{2}-(r(x)-t)^{2}\right]=\pi\left[2 r(x) t-t^{2}\right], W(x)=\frac{J(x)}{r(x)},
$$

are the cross sectional area and the elastic section modulus respectively, the $f_{\mathrm{y}}$ is the yield stress.

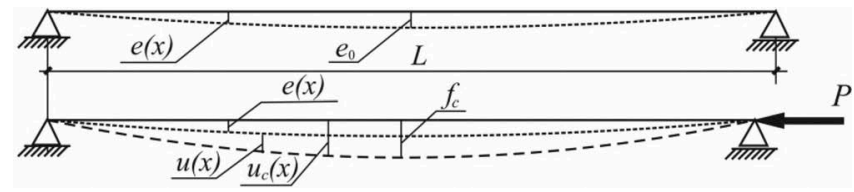

Figure. 4. Deflections of the initially curved rod. 
Using (5) and taking the equality in (6) we obtain:

$$
\frac{P}{A(x)}+\frac{P}{W(x)} e_{0} \frac{P_{k r}}{P_{k r}-P} \sin \frac{\pi x}{L}=f_{y}
$$

To convert the equation (8) to the form known from EN1993-1-1 (2005) let us introduce the notations:

$$
P(x)=\chi(x) \cdot A(x) \cdot f_{y}, \hat{\lambda}(x)=\sqrt{\frac{f_{y} A(x)}{P_{k r}}},
$$

where: $\chi(\mathrm{x})$ is the buckling reduction factor, $\hat{\lambda}(x)$ is the dimensionless measure of the slenderness, both dependent on $\mathrm{x}$ in this particular case.

Substituting (9) to eqn. (8) and introducing the quantity $\Phi(\mathrm{x})$ defined as follows:

$$
\Phi(x)=\frac{1}{2}\left[1+\frac{A(x)}{W(x)} e_{0} \sin \frac{\pi x}{L}+\hat{\lambda}^{2}(x)\right]
$$

one obtains the following equation:

$$
\chi^{2}(x) \cdot \hat{\lambda}^{2}(x)-\chi(x) \cdot 2 \Phi(x)+1=0
$$

from which the searched reduction factor $\chi(\mathrm{x})$ is obtained in the form:

$$
\chi(x)=\frac{\Phi(x)-\sqrt{\Phi^{2}(x)-\hat{\lambda}^{2}(x)}}{\hat{\lambda}^{2}(x)}=\frac{1}{\Phi(x)+\sqrt{\Phi^{2}(x)-\hat{\lambda}^{2}(x)}},
$$

which is consistent with the formula (6.49) from EN 1993-1-1 (2005) valid for columns of constant cross-section.

The column's resistance $P_{\text {ult }}$ is determined by the smallest value of the expression:

$$
P_{u l t}(x)=\chi(x) \cdot A(x) \cdot f_{y} .
$$

The value of ultimate force defined by formulae (13) could be too high in some circumstances. For save design procedures the additional partial coefficient $\gamma=1.2$ is proposed and the final formula for design value of column's resistance is as follows:

$$
P_{R d}(x)=\frac{1}{\gamma} \cdot \chi(x) \cdot A(x) \cdot f_{y}
$$

The whole procedure can be easily inserted in spreadsheet for the every $x$ from the interval $0<x<L / 2$, and in this way the smallest value of $P_{R d}$ can be found.

The initial bow amplitude $e_{0}$ required in this procedure can be adopted according to the code recommendations. Following provisions inserted in eurocodes specifying tolerances, dimensions and sectional properties of steel structural elements the $e_{0}$ can be adopted as $L / 750$ and this value guaranteeing the conservative assessment of columns resistance was adopted in examples presented in the next section. 


\section{EXAMPLES}

As the first example of the application of presented procedure let us consider the bulged, steel bar of length $L=1000 \mathrm{~mm}, D_{1}=L / 100=10 \mathrm{~mm}, D_{2}=2 L / 100=20 \mathrm{~mm}, t=L / 1000=1 \mathrm{~mm}$ and $e_{0}=L / 750=1.333 \mathrm{~mm}$. Material parameters: $E=210 \mathrm{GPa}$, the yield stress $f_{\mathrm{y}}=355 \mathrm{MPa}$.

The critical force is calculated by means of the formula (Table 1) is $P_{k r}=4.032 \mathrm{kN}$. Using the spreadsheet the sequence of $P_{\text {ult }}$ were calculated from the formula (13) for $x=0$ to 500 $(L / 2)$ with step $5 \mathrm{~mm}$. The smallest value of the compression force $P_{\text {ult, } \min }=3.766 \mathrm{kN}$ and this value was obtained for $x=220 \mathrm{~mm}$. The column's design resistance calculated from the formula (14) is equal $P_{\mathrm{Rd}}=3.138 \mathrm{kN}$ and it is the searched measure of the bar's compression resistance.

The same example was solved numerically by means of Abaqus system. Two kind of modelling were adopted in numerical simulations which were carried out and namely the shell model (63126 DOF) and the 3D (solid) model (94695 DOF). Using these models for $1 / 4$ of the column (the column had two planes of symmetry) the geometrically and materially nonlinear analysis with imperfections (GMNIA) was performed. The uniformly distributed load was applied at the columns end. The bilinear material model for steel was adopted. Equilibrium paths obtained by two models were nearly identical (less than $2 \%$ ) which confirms the correctness of the simulation performed.

The $\sigma_{x}=355 \mathrm{MPa}$ appeared at section $x=237.5 \mathrm{~mm}$ (comp. Figure 5) when the load attained value $P=3.874 \mathrm{kN}$. It is the value $2.5 \%$ higher than the result obtained with the analytical method. The maximum load on the equilibrium path was equal $P_{\max }=3.883 \mathrm{kN}$. The design value of column's resistance was equal $3.874 / 1.2=3.23 \mathrm{kN}$ and this level was shown in Figure 5.

As the other example let us consider the column of following parameters: $D_{2}=4 \mathrm{~L} /$ $100=40 \mathrm{~mm}, t=3 L / 1000=3 \mathrm{~mm}$. Other geometrical and material parameters were the same.

The critical force is calculated by means of the formula (Table 1) is $P_{k r}=62.447 \mathrm{kN}$. In this case the $P_{u l t \text { min }}=23.421 \mathrm{kN}$ and this value was obtained for $x=0 \mathrm{~mm}$ (column's end). The column's design resistance calculated from the formula (14) is equal $P_{R d}$ $=19.52 \mathrm{kN}$. The counterpart of this value obtained numerically was equal $18.51 \mathrm{kN}$.

In both examples the column's design resistances obtained analytically were conservative.

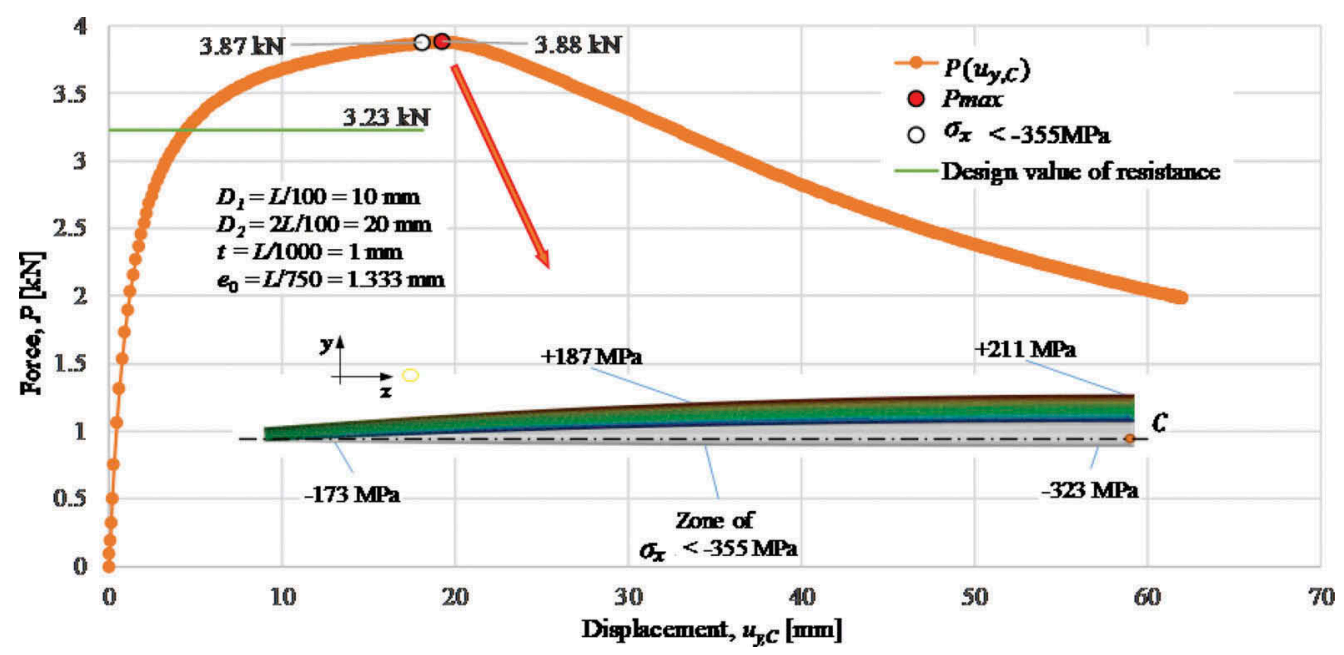

Figure 5. Nonlinear equilibrium path obtained in numerical simulation. 


\section{RECAPITULATION AND CONCLUSIONS}

Existing design regulations do not contain provisions for design of steel, compressed members of structures when they are non-prismatic. Authors have presented the procedure which allows determining the critical force for non-prismatic rods of specific geometry. Knowing the critical force one can assess the compression resistance of the rod under consideration using the other, based on Ayrton-Perry approach, procedure details of which were presented in the paper. Due to the fact that the cross section is variable, the stress criterion which follows from AyrtonPerry condition should be checked not only in middle section of the bar but also in all remaining cross-sections. This stage of the procedure can be accomplished easily by means of the spreadsheet in which formulae presented in this paper should be inserted for arbitrary value of the axial coordinate $x$ of the rod. The smallest value of compression force obtained as the result of the presented procedure is the measure of compression resistance of the considered rod.

Examples presented in the paper confirm that the proposed procedure is relatively easy, effective and correct what was proved in geometrically and materially nonlinear numerical simulations presented in the paper. This design procedure can be recommended for civil engineers designing steel, structural members of the particular shape considered by authors. The design value of compressive force obtained as a final result of the procedure can be treated as a compressive resistance of analysed columns. The presented proposal effectively fills the gap existing in the design recommendations.

\section{REFERENCES}

Abaqus Documentation. 2020 - Massachusetts Institute Of Technology. Available online: https://abaqusdocs.mit.edu/2017/English/SIMACAEMATRefMap/simaexc-c-docproc.htm (accessed on 3.03.2020)

Ayrton, W.E. \& Perry, J. 1886. On struts, The Engineer 62: 464 465.

COSMOS/M. 2002. Finite Element Analysis System, Version 2.9, Structural Research and Analysis Corporation, Electronic Manual, Los Angeles, California.

EN 1993-1-1:2005, Eurocode 3: Design of steel structures - Part 1-1: General rules and rules for buildings, CEN, Brussels 2005.

Elishakoff, I. \& Rollot, O. 1999. New closed-form solutions for buckling of a variable stiffness column by mathematica, Journal of Sound and Vibration 224 (1): 172-82.

Giżejowski, M. A. \& Stachura, Z. \& Szczerba, R. B. \& Gajewski M. D. 2019. Buckling resistance of steel H-section beam-columns: In-plane buckling resistance, Journal of Constructional Steel Research 157 (June): 347-358.

Li, Q.S. \& Cao, H. \& Li, G. 1995. Stability analysis of bars with varying cross-section, International Journal of Solids and Structures, 32 (21): 3217-3228.

Li, Q.S. 2001. Exact solutions for buckling of non-uniform columns under axial concentrated and distributed loading, European Journal of Mechanics - A/Solids 20 (3): 485-500.

Marcinowski, J. 2015. Maximum elastic buckling resistance of columns of constant volume. XIV Stability of structures XIV-th Symposium, 08-12 June 2015, Zakopane.

Marcinowski, J. \& Sadowski, M. Using the ERFI Function in the Problem of the Shape Optimization of the Compressed Rod, International Journal of Automotive and Mechanical Engineering 25 (2): 75-87. DOI: 10.2478/ijame-2020-0021.

Autodesk Robot Structural Analysis Professional 2016. User's manual.

Szalai, J. \& Papp, F. 2010. On the theoretical background of the generalization of Ayrton-Perry type resistance formulas, Journal of Constructional Steel Research 66 (5) May: 670-679.

Szalai, J. 2017. Complete generalization of the Ayrton-Perry formula for beam-column buckling problems, Engineering Structures 153 (15) December: 205-223.

Timoshenko, S. \& Gere, J. 1961. Theory of elastic stability. New York: McGraw-Hill.

Thompson, P. \& Papadopoulou, G. \& Vassiliou, E. 2007. The origins of entasis: illusion, aesthetics or engineering?, Spatial Vision 20 (6):531-543.

Wolfram, S. 1988. Mathematica ${ }^{\mathrm{TM}}$, A system for Doing Mathematics by Computer.: Addison-Wesley Publishing Company, Inc. 